\title{
ON THE STABILITY OF GENERALIZED D'ALEMBERT AND JENSEN FUNCTIONAL EQUATIONS
}

\author{
GWANG HUI KIM AND SEVER S. DRAGOMIR
}

Received 19 March 2006; Revised 19 July 2006; Accepted 25 July 2006

The aim of this paper is to study the stability problem of the generalized d'Alembert, Wilson, and Jensen functional equations.

Copyright (c) 2006 Hindawi Publishing Corporation. All rights reserved.

\section{Introduction}

Baker et al. [4] and Bourgin [5] introduced that if $f$ satisfies the inequality $\mid E_{1}(f)-$ $E_{2}(f) \mid \leq \varepsilon$, then either $f$ is bounded or $E_{1}(f)=E_{2}(f)$. Thereafter it is called the superstability. Baker [3] proved the superstability of the cosine functional equation

$$
f(x+y)+f(x-y)=2 f(x) f(y)
$$

which is also called the d'Alembert functional equation.

The d'Alembert functional equation (A) is generalized to the following functional equations:

$$
\begin{aligned}
& f(x+y)+f(x-y)=2 f(x) g(y), \\
& f(x+y)+f(x-y)=2 g(x) f(y),
\end{aligned}
$$

where $f, g$ are two unknown functions to be determined.

Equation $\left(\mathrm{A}_{f g}\right)$, raised by Wilson, is called the Wilson equation sometimes, and $\left(\mathrm{A}_{g f}\right)$ is raised by Kannappan and $\mathrm{Kim}[9]$.

Let $g(x) \equiv k$ in $\left(\mathrm{A}_{g f}\right)$. Then we have $f(x+y)+f(x-y)=2 k f(y)$ for all $x, y \in G$. Putting $y=0$ in this equation we have $f(x)=k f(0)$. Hence $f$ is a constant function.

Let $g(y) \equiv 1$ in $\left(\mathrm{A}_{f g}\right)$. Then we have the Jensen functional equation

$$
f(x+y)+f(x-y)=2 f(x) .
$$

Hindawi Publishing Corporation

International Journal of Mathematics and Mathematical Sciences

Volume 2006, Article ID 43185, Pages 1-12

DOI 10.1155/IJMMS/2006/43185 
2 Stability of generalized d'Alembert and Jensen equations

Equations $(\mathrm{A}),\left(\mathrm{A}_{f g}\right)$, and $(\mathrm{J})$ has been investigated by Badora, Ger, Kannappan, Kim, $\mathrm{Ng}$, Sinopoulos, Stetkær, Székelyhidi, Yang, and so forth [1, 2, 6, 9-15].

The superstability of these equations $\left(\mathrm{A}_{f g}\right),\left(\mathrm{A}_{g f}\right)$ was investigated in $[9,10,14]$.

Given mappings $f, g: G \rightarrow \mathbb{C}$, we define a difference operator $D A: G \times G \rightarrow \mathbb{C}$ as

$$
D A(x, y):=f(x+y)+f(x-y)-2 f(x) f(y)
$$

which is called the approximate remainder of the functional equation (A) and acts as a perturbation of the equation.

Badora and Ger [2] proved the superstability of the d'Alembert equation (A) under the condition $|D A(x, y)| \leq \varphi(x)$ or $\varphi(y)$.

The aim of this paper is to investigate the improved superstability for functional equations $\left(\mathrm{A}_{f g}\right),\left(\mathrm{A}_{g f}\right)$, and $(\mathrm{J})$ as follows:

$$
\begin{array}{ccc}
\left|D A_{f g}(x, y)\right| \leq \varphi(x) & \text { or } & \varphi(y), \\
\left|D A_{g f}(x, y)\right| \leq \varphi(x) & \text { or } & \varphi(y), \\
|D J(x, y)| \leq \varphi(x, y) . &
\end{array}
$$

In this paper, let $(G,+)$ be an Abelian group, $\mathbb{C}$ the field of complex numbers, and $\mathbb{R}$ the field of real numbers. We may assume that $f$ and $g$ are nonzero functions and $\varepsilon$ is a nonnegative real constant, $\varphi: G \rightarrow \mathbb{R}$. If in all the results of this article we consider the Kannappan condition $f(x+y+z)=f(x+z+y)$ in [8], then we will obtain identical results for the semigroup $(G,+)$.

\section{Stability of $\left(\mathbf{A}_{f g}\right)$ and $\left(\mathbf{A}_{g f}\right)$}

In this section, we will investigate the stability of the generalized d'Alembert functional equations $\left(\mathrm{A}_{f g}\right)$ and $\left(\mathrm{A}_{g f}\right)$ related to the d'Alembert functional equation $(\mathrm{A})$.

Theorem 2.1. Suppose that $f, g: G \rightarrow \mathbb{C}$ satisfy the inequality

$$
|f(x+y)+f(x-y)-2 f(x) g(y)| \leq\left\{\begin{array}{l}
(\mathrm{i}) \varphi(y) \\
(\text { ii) } \varphi(x), \varphi(y)
\end{array}\right.
$$

for all $x, y \in G$; and in case (ii), assume that $f(-x)=f(x)$, for $x \in G$.

Then

(i) either $f$ is bounded or $g$ satisfies (A),

(ii) either $g($ or $f)$ is bounded or $g$ satisfies $(A)$, also $f$ and $g$ satisfy $\left(A_{f g}\right)$ and $\left(A_{g f}\right)$.

Proof. For the case (i), let $f$ be unbounded. Then we can choose a sequence $\left\{x_{n}\right\}$ in $G$ such that

$$
0 \neq\left|f\left(x_{n}\right)\right| \longrightarrow \infty \text { as } n \longrightarrow \infty
$$


we will show that $g$ satisfies (A). Taking $x=x_{n}$ in (2.1) we obtain

$$
\left|\frac{f\left(x_{n}+y\right)+f\left(x_{n}-y\right)}{2 f\left(x_{n}\right)}-g(y)\right| \leq \frac{\varphi(y)}{\left|2 f\left(x_{n}\right)\right|},
$$

that is,

$$
\lim _{n \rightarrow \infty} \frac{f\left(x_{n}+y\right)+f\left(x_{n}-y\right)}{2 f\left(x_{n}\right)}=g(y), \quad y \in G .
$$

Using (i) of (2.1) we have

$$
\begin{aligned}
& \mid f\left(\left(x_{n}+x\right)+y\right)+f\left(\left(x_{n}+x\right)-y\right)-2 f\left(x_{n}+x\right) g(y) \\
& \quad+f\left(\left(x_{n}-x\right)+y\right)+f\left(\left(x_{n}-x\right)-y\right)-2 f\left(x_{n}-x\right) g(y) \mid \leq 2 \varphi(y),
\end{aligned}
$$

so that

$$
\begin{aligned}
& \mid \frac{f\left(x_{n}+(x+y)\right)+f\left(x_{n}-(x+y)\right)}{2 f\left(x_{n}\right)} \\
& \quad+\frac{f\left(x_{n}+(x-y)\right)+f\left(x_{n}-(x-y)\right)}{2 f\left(x_{n}\right)}-2 \frac{f\left(x_{n}+x\right)+f\left(x_{n}-x\right)}{2 f\left(x_{n}\right)} g(y) \mid \leq \frac{\varphi(y)}{\left|f\left(x_{n}\right)\right|}
\end{aligned}
$$

for all $x, y \in G$. By virtue of (2.2) and (2.4), we have

$$
|g(x+y)+g(x-y)-2 g(x) g(y)| \leq 0
$$

for all $x, y \in G$. Therefore $g$ satisfies (A).

For the case (ii), first we show that $f$ is unbounded if and only if $g$ is also unbounded.

Namely, if $f$ is bounded, choose $x_{0} \in G$ such that $f\left(x_{0}\right) \neq 0$ and use (ii) of (2.1) to get

$$
|g(y)|-\frac{\left|f\left(x_{0}+y\right)+f\left(x_{0}-y\right)\right|}{2\left|f\left(x_{0}\right)\right|} \leq\left|\frac{f\left(x_{0}+y\right)+f\left(x_{0}-y\right)}{2 f\left(x_{0}\right)}-g(y)\right| \leq \frac{\varphi\left(x_{0}\right)}{2\left|f\left(x_{0}\right)\right|},
$$

which shows that $g$ is also bounded.

Suppose $f$ is unbounded. Putting $x=0$ in (ii) of (2.1), we have

$$
|f(y)+f(-y)-2 f(0) g(y)| \leq \varphi(0),
$$

that is,

$$
|f(y)-f(0) g(y)| \leq \frac{\varphi(0)}{2}
$$

since $f(-x)=f(x)$ for all $x \in G$. This implies that $g$ is also unbounded.

Let $g$ be unbounded, then $f$ is also unbounded. Then we can choose sequences $\left\{x_{n}\right\}$ and $\left\{y_{n}\right\}$ in $G$ such that $f\left(x_{n}\right) \neq 0$ and $\left|f\left(x_{n}\right)\right| \rightarrow \infty, g\left(y_{n}\right) \neq 0$ and $\left|g\left(y_{n}\right)\right| \rightarrow \infty$ as $n \rightarrow \infty$. 
4 Stability of generalized d'Alembert and Jensen equations

Taking $y=y_{n}$ in (ii) of (2.1) we deduce

$$
\lim _{n \rightarrow \infty} \frac{f\left(x+y_{n}\right)+f\left(x-y_{n}\right)}{2 g\left(y_{n}\right)}=f(x)
$$

for all $x \in G$. Again applying (ii) of (2.1) we have

$$
\begin{aligned}
& \mid f\left(x+\left(y+y_{n}\right)\right)+f\left(x-\left(y+y_{n}\right)\right)-2 f(x) g\left(y+y_{n}\right) \\
& \quad+f\left(x+\left(y-y_{n}\right)\right)+f\left(x-\left(y-y_{n}\right)\right)-2 f(x) g\left(y-y_{n}\right) \mid \leq 2 \varphi(x),
\end{aligned}
$$

so that

$$
\begin{aligned}
& \mid \frac{f\left((x+y)+y_{n}\right)+f\left((x+y)-y_{n}\right)}{2 g\left(y_{n}\right)} \\
& \quad+\frac{f\left((x-y)+y_{n}\right)+f\left((x-y)-y_{n}\right)}{2 g\left(y_{n}\right)}-2 f(x) \frac{g\left(y_{n}+y\right)+g\left(y_{n}-y\right)}{2 g\left(y_{n}\right)} \mid \\
& \quad \leq \frac{\varphi(x)}{\left|g\left(y_{n}\right)\right|}
\end{aligned}
$$

for all $x, y \in G$. Since $g$ satisfies (A) by (i), it follows from (2.11) that

$$
|f(x+y)+f(x-y)-2 f(x) g(y)| \leq 0
$$

for all $x, y \in G$. Hence $f$ and $g$ are solutions of $\left(\mathrm{A}_{f g}\right)$.

Using (ii) of (2.1) we have

$$
\begin{aligned}
& \mid f\left(y+\left(x+y_{n}\right)\right)+f\left(y-\left(x+y_{n}\right)\right)-2 f(y) g\left(x+y_{n}\right) \\
& \quad+f\left(y+\left(x-y_{n}\right)\right)+f\left(y-\left(x-y_{n}\right)\right)-2 f(y) g\left(x-y_{n}\right) \mid \leq 2 \varphi(y)
\end{aligned}
$$

for all $x, y \in G$. Since $f(-x)=f(x)$ for all $x \in G$, we have

$$
\begin{aligned}
& \mid \frac{f\left((x+y)+y_{n}\right)+f\left((x+y)-y_{n}\right)}{2 g\left(y_{n}\right)} \\
& \quad+\frac{f\left((x-y)+y_{n}\right)+f\left((x-y)-y_{n}\right)}{2 g\left(y_{n}\right)}-2 f(y) \frac{g\left(y_{n}+x\right)+g\left(y_{n}-x\right)}{2 g\left(y_{n}\right)} \mid \\
& \quad \leq \frac{\varphi(y)}{\left|g\left(y_{n}\right)\right|}
\end{aligned}
$$

for all $x, y \in G$. Since $g$ satisfies (A), using (2.11), we have

$$
|f(x+y)+f(x-y)-2 g(x) f(y)| \leq 0
$$

for all $x, y \in G$. Therefore $f$ and $g$ are solutions of $\left(\mathrm{A}_{g f}\right)$, which ends the proof. 
Corollary $2.2[9,14]$. Suppose that $f, g: G \rightarrow \mathbb{C}$ satisfy the inequality

$$
|f(x+y)+f(x-y)-2 f(x) g(y)| \leq \varepsilon
$$

for all $x, y \in G$. Then

(i) either $f$ is bounded or $g$ satisfies $(A)$,

(ii) under the assumption $f(-x)=f(x)$ for $x \in G$, either $g$ (or $f$ ) is bounded or $g$ satisfies $(A)$, also $f$ and $g$ satisfy $\left(A_{f g}\right)$ and $\left(A_{g f}\right)$.

Corollary 2.3 [2]. Suppose that $f: G \rightarrow \mathbb{C}$ satisfies the inequality

$$
|f(x+y)+f(x-y)-2 f(x) f(y)| \leq \varphi(y)
$$

for all $x, y \in G$. Then either $f$ is bounded or $f$ satisfies (A).

Corollary 2.4 [3]. Suppose that $f: G \rightarrow \mathbb{C}$ satisfies the inequality

$$
|f(x+y)+f(x-y)-2 f(x) f(y)| \leq \varepsilon
$$

for all $x, y \in G$. Then either $f$ is bounded or $f$ satisfies $(A)$.

Theorem 2.5. Suppose that $f, g: G \rightarrow \mathbb{C}$ satisfy the inequality

$$
|f(x+y)+f(x-y)-2 g(x) f(y)| \leq\left\{\begin{array}{l}
(\mathrm{i}) \varphi(x), \\
(\text { ii) } \varphi(y), \varphi(x)
\end{array}\right.
$$

for all $x, y \in G$. Then

(i) either $f$ is bounded or $g$ satisfies (A),

(ii) either $g($ or $f)$ is bounded or $g$ satisfies $(A)$, also $f$ and $g$ satisfy $\left(A_{g f}\right)$ and $\left(A_{f g}\right)$.

Proof. In the case (i), for the unbounded $f$, we can choose a sequence $\left\{y_{n}\right\}$ in $G$ such that $\left|f\left(y_{n}\right)\right| \rightarrow \infty$ as $n \rightarrow \infty$ like (2.2) of Theorem 2.1.

Taking $y=y_{n}$ in (2.21) we obtain

$$
\left|\frac{f\left(x+y_{n}\right)+f\left(x-y_{n}\right)}{2 f\left(y_{n}\right)}-g(x)\right| \leq \frac{\varphi(x)}{2\left|f\left(y_{n}\right)\right|},
$$

that is,

$$
\lim _{n \rightarrow \infty} \frac{f\left(x+y_{n}\right)+f\left(x-y_{n}\right)}{2 f\left(y_{n}\right)}=g(x)
$$

for all $x \in G$. Using (i) of (2.21) we have

$$
\begin{aligned}
& \mid f\left(x+\left(y+y_{n}\right)\right)+f\left(x-\left(y+y_{n}\right)\right)-2 g(x) f\left(y+y_{n}\right) \\
& +f\left(x+\left(y-y_{n}\right)\right)+f\left(x-\left(y-y_{n}\right)\right)-2 g(x) f\left(y-y_{n}\right) \mid \leq 2 \varphi(x)
\end{aligned}
$$


6 Stability of generalized d'Alembert and Jensen equations

so that

$$
\begin{aligned}
& \mid \frac{f\left((x+y)+y_{n}\right)+f\left((x+y)-y_{n}\right)}{2 f\left(y_{n}\right)} \\
& \quad+\frac{f\left((x-y)+y_{n}\right)+f\left((x-y)-y_{n}\right)}{2 f\left(y_{n}\right)}-2 g(x) \frac{f\left(y+y_{n}\right)+f\left(y-y_{n}\right)}{2 f\left(y_{n}\right)} \mid \\
& \quad \leq \frac{\varphi(x)}{\left|f\left(y_{n}\right)\right|}
\end{aligned}
$$

for all $x, y \in G$. By virtue of $\left|f\left(y_{n}\right)\right| \rightarrow \infty$ and (2.23), we have

$$
|g(x+y)+g(x-y)-2 g(x) g(y)| \leq 0
$$

for all $x, y \in G$. Therefore $g$ satisfies (A).

For the proof of the case (ii), we can see that, similar to Theorem 2.1, $f$ is unbounded if and only if $g$ is also unbounded. Namely, putting $y=0$ in (ii) of (2.21) we obtain

$$
|f(x)-g(x) f(0)| \leq \frac{\varphi(0)}{2}
$$

for all $x \in G$. If $g$ is bounded, then by (2.27), we have

$$
|f(x)|=|f(x)-g(x) f(0)+g(x) f(0)| \leq \frac{\varphi(0)}{2}+|g(x) f(0)|,
$$

which shows that $f$ is also bounded. On the other hand if $f$ is bounded, we choose $y_{0} \in G$ such that $f\left(y_{0}\right) \neq 0$, and then by (2.21) we obtain

$$
|g(x)|-\left|\frac{f\left(x+y_{0}\right)+f\left(x-y_{0}\right)}{2 f\left(y_{0}\right)}\right| \leq\left|\frac{f\left(x+y_{0}\right)+f\left(x-y_{0}\right)}{2 f\left(y_{0}\right)}-g(x)\right| \leq \frac{\varphi\left(y_{0}\right)}{2\left|f\left(y_{0}\right)\right|}
$$

and it follows that $g$ is also bounded on $G$.

Namely, if $f$ (or $g$ ) is unbounded, then so is $g$ ( or $f$ ).

Let $g$ be unbounded, then $f$ is also unbounded. Then we can choose sequences $\left\{x_{n}\right\}$ and $\left\{y_{n}\right\}$ in $G$ such that $g\left(x_{n}\right) \neq 0$ and $\left|g\left(x_{n}\right)\right| \rightarrow \infty, f\left(y_{n}\right) \neq 0$ and $\left|f\left(y_{n}\right)\right| \rightarrow \infty$ as $n \rightarrow \infty$.

Taking $x=x_{n}$ in (ii) of (2.21) we deduce

$$
\lim _{n \rightarrow \infty} \frac{f\left(x_{n}+y\right)+f\left(x_{n}-y\right)}{2 g\left(x_{n}\right)}=f(y)
$$

for all $y \in G$. Using (2.21) we have

$$
\begin{aligned}
& \mid f\left(\left(x_{n}+x\right)+y\right)+f\left(\left(x_{n}+x\right)-y\right)-2 g\left(x_{n}+x\right) f(y) \\
& +f\left(\left(x_{n}-x\right)+y\right)+f\left(\left(x_{n}-x\right)-y\right)-2 g\left(x_{n}-x\right) f(y) \mid \leq 2 \varphi(y)
\end{aligned}
$$


for all $x, y \in G$ and every $n \in \mathbb{N}$. Consequently,

$$
\begin{aligned}
& \mid \frac{f\left(x_{n}+(x+y)\right)+f\left(x_{n}-(x+y)\right)}{2 g\left(x_{n}\right)} \\
& \quad+\frac{f\left(x_{n}+(x-y)\right)+f\left(x_{n}-(x-y)\right)}{2 g\left(x_{n}\right)}-2 \cdot \frac{g\left(x_{n}+x\right)+g\left(x_{n}-x\right)}{2 g\left(x_{n}\right)} f(y) \mid \\
& \quad \leq \frac{\varphi(y)}{\left|g\left(x_{n}\right)\right|}
\end{aligned}
$$

for all $x, y \in G$ and every $n \in \mathbb{N}$. Passing here to the limit as $n \rightarrow \infty$ with the use of $\left|g\left(x_{n}\right)\right| \rightarrow \infty$ and (2.30). Since $g$ satisfies (A) by (i), $f$ and $g$ are solutions of $\left(\mathrm{A}_{g f}\right)$.

Applying (ii) of (2.21) again, we get

$$
\begin{aligned}
& \mid f\left(\left(x_{n}+y\right)+x\right)+f\left(\left(x_{n}+y\right)-x\right)-2 g\left(x_{n}+y\right) f(x) \\
& +f\left(\left(x_{n}-y\right)+x\right)+f\left(\left(x_{n}-y\right)-x\right)-2 g\left(x_{n}-y\right) f(x) \mid \leq 2 \varphi(x), \\
& \mid \frac{f\left(x_{n}+(x+y)\right)+f\left(x_{n}-(x+y)\right)}{2 g\left(x_{n}\right)} \\
& \quad+\frac{f\left(x_{n}+(x-y)\right)+f\left(x_{n}-(x-y)\right)}{2 g\left(x_{n}\right)}-2 f(x) \cdot \frac{g\left(x_{n}+y\right)+g\left(x_{n}-y\right)}{2 g\left(x_{n}\right)} \mid \\
& \quad \leq \frac{\varphi(x)}{\left|g\left(x_{n}\right)\right|}
\end{aligned}
$$

for all $x, y \in G$ and every $n \in \mathbb{N}$.

Using (2.30) and the fact that $g$ satisfies (A) by (i), the last inequality yields that $f$ and $g$ are solutions of $\left(\mathrm{A}_{f g}\right)$.

Corollary 2.6 $[9,14]$. Suppose that $f, g: G \rightarrow \mathbb{C}$ satisfy the inequality

$$
|f(x+y)+f(x-y)-2 g(x) f(y)| \leq \varepsilon
$$

for all $x, y \in G$. Then

(i) either $f$ is bounded or $g$ satisfies (A),

(ii) either $g($ or $f)$ is bounded or $g$ satisfies $(A)$, also $f$ and $g$ satisfy $\left(A_{g f}\right)$ and $\left(A_{f g}\right)$.

Corollary $2.7[2]$. Suppose that $f, g: G \rightarrow \mathbb{C}$ satisfy the inequality

$$
|f(x+y)+f(x-y)-2 f(x) f(y)| \leq \varphi(x)
$$

for all $x, y \in G$. Then either $f$ is bounded or $f$ satisfies $(A)$.

Remark 2.8. Let $f, g: \mathbb{R} \rightarrow \mathbb{R}$ be functions with $f(x)=x$ and $g(x) \equiv 1$ for all $x \in \mathbb{R}$. Then we know that $|f(x+y)+f(x-y)-2 f(x) g(y)|=0$, but $f$ is unbounded and $f, g$ do not satisfy $\left(\mathrm{A}_{g f}\right)$, that is, $|f(x+y)+f(x-y)-2 g(x) f(y)| \neq 0$. This shows that the condition $f(-x)=f(x)$ is essential in case (ii) of Theorem 2.1.

All results obtained can be extended to the superstability on the Banach algebra. To simplify, we will combine two theorems in one. 
8 Stability of generalized d'Alembert and Jensen equations

Theorem 2.9. Let $(E,\|\cdot\|)$ be a semisimple commutative Banach algebra. Assume that $f, g: G \rightarrow E$ and $\varphi: G \rightarrow \mathbb{R}$ satisfy one of the inequalities

$$
\|f(x+y)+f(x-y)-2 f(x) g(y)\| \leq\left\{\begin{array}{l}
(\text { i) } \varphi(y), \\
(\text { ii) } \varphi(x), \varphi(y),
\end{array} \quad x, y \in G\right.
$$

or

$$
\|f(x+y)+f(x-y)-2 g(x) f(y)\| \leq\left\{\begin{array}{l}
(\text { i) } \varphi(x), \\
\text { (ii) } \varphi(y), \varphi(x),
\end{array} \quad x, y \in G\right.
$$

with $f(-x)=f(x)$ in case (ii) of (2.36).

(a) If the superposition $x^{*} \circ f$ is unbounded for each linear multiplicative functional $x^{*} \in E^{*}$, then $g$ satisfies ( $A$ ) in each case (i) of (2.36) and (2.37).

(b) If the superposition $x^{*} \circ g$ is unbounded for each linear multiplicative functional $x^{*} \in$ $E^{*}$, then $g$ satisfies $(A)$ and also $f$ and $g$ satisfy $\left(A_{f g}\right)$ and $\left(A_{g f}\right)$ in each case (ii) of (2.36) and (2.37).

Proof. The proofs of each case are very similar, so it suffices to show the proof of case (ii) of (2.36) in (b). Assume that (ii) of (2.36) holds and fix arbitrarily a linear multiplicative functional $x^{*} \in E$. As is well known we have $\left\|x^{*}\right\|=1$, whence, for every $x, y \in G$, we have

$$
\begin{aligned}
\varphi(x) & \geq\|f(x+y)+f(x-y)-2 f(x) g(y)\| \\
& =\sup _{\left\|y^{*}\right\|=1}\left|y^{*}(f(x+y)+f(x-y)-2 f(x) g(y))\right| \\
& \geq\left|x^{*}(f(x+y))+x^{*}(f(x-y))-2 x^{*}(f(x)) x^{*}(g(y))\right|,
\end{aligned}
$$

which states that the superpositions $x^{*} \circ f$ and $x^{*} \circ g$ yield a solution of inequality (ii) of (2.1) in Theorem 2.1. Since, by assumption, the superposition $x^{*} \circ g$ is unbounded with $f(-x)=f(x)$, an appeal to Theorem 2.1 shows that the functions $x^{*} \circ f$ and $x^{*} \circ g$ solve $\left(\mathrm{A}_{f g}\right)$. In other words, bearing the linear multiplicativity of $x^{*}$ in mind, for all $x, y \in G$, the difference $A_{f g}(x, y)$ falls into the kernel of $x^{*}$. Therefore, in view of the unrestricted choice of $x^{*}$, we infer that

$$
A_{f g}(x, y) \in \bigcap\left\{\operatorname{ker} x^{*}: x^{*} \text { is a multiplicative member of } E^{*}\right\}
$$

for all $x, y \in G$. Since the algebra $E$ has been assumed to be semisimple, the last term of the above formula coincides with the singleton $\{0\}$, that is,

$$
f(x+y)+f(x-y)-2 f(x) g(y)=0 \quad \forall x, y \in G,
$$

as claimed. The other cases are similar.

Considering cases $g=f$ and $\varphi(y)=\varphi(x)=\varepsilon$, we can get additional corollaries. 
Corollary 2.10. Let $(E,\|\cdot\|)$ be a semisimple commutative Banach algebra. Assume that $f, g: G \rightarrow$ E satisfy one of the inequalities

$$
\|f(x+y)+f(x-y)-2 f(x) g(y)\| \leq \varepsilon, \quad x, y \in G,
$$

with $f(-x)=f(x)$ or

$$
\|f(x+y)+f(x-y)-2 g(x) f(y)\| \leq \varepsilon, \quad x, y \in G .
$$

(a) If the superposition $x^{*} \circ f$ is unbounded for each linear multiplicative functional $x^{*} \in E^{*}$, then $g$ satisfies $(A)$ in each case.

(b) If the superposition $x^{*} \circ g$ is unbounded for each linear multiplicative functional $x^{*} \in$ $E^{*}$, then $g$ satisfies $(A)$ and also $f$ and $g$ satisfy $\left(A_{f g}\right)$ and $\left(A_{g f}\right)$ in each case.

Corollary 2.11. Let $(E,\|\cdot\|)$ be a semisimple commutative Banach algebra. Assume that $f: G \rightarrow E$ and $\varphi: G \rightarrow \mathbb{R}$ satisfy one of the inequalities

$$
\|f(x+y)+f(x-y)-2 f(x) f(y)\| \leq\left\{\begin{array}{l}
(\text { i) } \varphi(y), \\
\text { (ii) } \varphi(x),
\end{array} \quad x, y \in G .\right.
$$

Then either the superposition $x^{*} \circ f$ is unbounded for each linear multiplicative functional $x^{*} \in E^{*}$, or $f$ satisfies $(A)$ in each case.

Corollary 2.12. Let $(E,\|\cdot\|)$ be a semisimple commutative Banach algebra. Assume that $f: G \rightarrow$ E satisfy one of the inequalities

$$
\|f(x+y)+f(x-y)-2 f(x) f(y)\| \leq \varepsilon, \quad x, y \in G
$$

Then either the superposition $x^{*} \circ f$ is unbounded for each linear multiplicative functional $x^{*} \in E^{*}$, or $f$ satisfies $(A)$.

\section{A solution and stability of the Jensen functional equation}

In this section, we prove the stability in the sense of Găvruţa [7] for Jensen functional equation $(\mathrm{J})$. We show that a general solution of $(\mathrm{J})$ is represented by a summation of the additive mapping and a constant.

Theorem 3.1. Let E be a Banach space. Suppose that $f: G \rightarrow$ E satisfies the inequality

$$
\|f(x+y)+f(x-y)-2 f(x)\| \leq \psi(x, y), \quad x, y \in G,
$$

where $\psi$ satisfies $\Psi(x, y):=\sum_{k=1}^{\infty}\left(1 / 2^{k}\right) \psi\left(2^{k-1} x, 2^{k-1} y\right)<\infty$ for $x, y \in G$.

Then there exists a unique additive mapping $A: G \rightarrow E$ as a solution of $(J)$ such that $A(-x)=-A(x)$ and

$$
\|f(x)-f(0)-A(x)\| \leq \Psi(x, x)
$$

for all $x \in G$. 
10 Stability of generalized d'Alembert and Jensen equations

Proof. Putting $y=x$ in (3.1) we have

$$
\|f(2 x)+f(0)-2 f(x)\| \leq \psi(x, x)
$$

for all $x \in G$. Let $F(x):=f(x)-f(0)$ for all $x \in G$. Then $F(0)=0$ and

$$
\|F(2 x)-2 F(x)\| \leq \psi(x, x)
$$

for all $x \in G$. Replacing $x$ by $2^{n} x$ in (3.4) and dividing its result by $2^{n+1}$ we get

$$
\left\|\frac{F\left(2^{n} x\right)}{2^{n}}-\frac{F\left(2^{n+1} x\right)}{2^{n+1}}\right\| \leq \frac{1}{2^{n+1}} \cdot \psi\left(2^{n} x, 2^{n} x\right)
$$

for all $x \in E$ and all nonnegative integers $n$. Using (3.5) and the triangle inequality we have

$$
\left\|\frac{F\left(2^{m} x\right)}{2^{m}}-\frac{F\left(2^{n} x\right)}{2^{n}}\right\| \leq \sum_{k=m+1}^{n} \frac{1}{2^{k}} \cdot \psi\left(2^{k-1} x, 2^{k-1} x\right)
$$

for all $x \in E$ and all nonnegative integers $m$ and $n$ with $m<n$. This shows that $\left\{F\left(2^{n} x\right) / 2^{n}\right\}$ is a Cauchy sequence for all $x \in E$ since the right side of (3.6) converges to zero by the assumption of $\varphi$ when $m \rightarrow \infty$. Consequently, we can define a mapping $A: G \rightarrow E$ by

$$
A(x):=\lim _{n \rightarrow \infty} \frac{F\left(2^{n} x\right)}{2^{n}}
$$

for all $x \in G$. Putting $m=0$ in (3.6) and taking the limit as $n \rightarrow \infty$, we obtain (3.2). Also, we get $A(0)=0$ and

$$
\begin{aligned}
\| A(x & +y)+A(x-y)-2 A(x) \| \\
& =\lim _{n \rightarrow \infty}\left\|\frac{F\left(2^{n} x+2^{n} y\right)}{2^{n}}+\frac{F\left(2^{n} x-2^{n} y\right)}{2^{n}}-2 \frac{F\left(2^{n} x\right)}{2^{n}}\right\| \\
& =\lim _{n \rightarrow \infty} \frac{1}{2^{n}}\left\|f\left(2^{n} x+2^{n} y\right)+f\left(2^{n} x-2^{n} y\right)-2 f\left(2^{n} x\right)\right\| \\
& \leq \lim _{n \rightarrow \infty} 2 \cdot \frac{1}{2^{n+1}} \psi\left(2^{n} x, 2^{n} y\right)=0
\end{aligned}
$$

for all $x, y \in G$, which means that $A$ satisfies (J). It also follows that $A$ is additive.

Now, let $A^{\prime}: G \rightarrow E$ be another additive mapping satisfying (3.2). Then we have

$$
\begin{aligned}
\| A(x) & -A^{\prime}(x) \| \\
& =2^{-n}\left\|A\left(2^{n} x\right)-A^{\prime}\left(2^{n} x\right)\right\| \\
& \leq 2^{-n}\left(\left\|A\left(2^{n} x\right)-f\left(2^{n} x\right)+f(0)\right\|+\left\|A^{\prime}\left(2^{n} x\right)-f\left(2^{n} x\right)+f(0)\right\|\right) \\
& \leq 2 \cdot \sum_{k=n+1}^{\infty} \frac{1}{2^{k}} \cdot \psi\left(2^{k-1} x, 2^{k-1} x\right)
\end{aligned}
$$


for all $x \in E$ and all positive integers $n$. Taking the limit in (3.9) as $n \rightarrow \infty$, we can conclude that $A(x)=A^{\prime}(x)$ for all $x \in E$. This proves the uniqueness of $A$.

Corollary 3.2. Let $E$ be a Banach space. Suppose that $f: G \rightarrow$ E satisfies the inequality

$$
\|f(x+y)+f(x-y)-2 f(x)\| \leq \varphi(x), \quad x, y \in G
$$

where $\varphi$ satisfies $\Phi(x):=\sum_{k=1}^{\infty}\left(1 / 2^{k}\right) \varphi\left(2^{k-1} x\right)<\infty$ for $x \in G$. Then there exists a unique additive mapping $A: G \rightarrow E$ as a solution of $(J)$ such that $A(-x)=-A(x)$ and

$$
\|f(x)-f(0)-A(x)\| \leq \Phi(x)
$$

for all $x \in E$.

Proof. Putting $\psi(x, y)=\varphi(x)$ in inequality (3.1), then it implies (3.10). The proof runs along the same procedure as Theorem 3.1.

Corollary 3.3. Let $E$ be a Banach space. Suppose that $f: G \rightarrow$ E satisfies the inequality

$$
\|f(x+y)+f(x-y)-2 f(x)\| \leq \varphi(y), \quad x, y \in G
$$

where $\varphi$ satisfies $\Phi(y):=\sum_{k=1}^{\infty}\left(1 / 2^{k}\right) \varphi\left(2^{k-1} y\right)<\infty$ for $y \in G$.

Then there exists a unique additive mapping $A: G \rightarrow E$ as a solution of $(J)$ such that $A(-x)=-A(x)$ and

$$
\|f(x)-f(0)-A(x)\| \leq \Phi(x)
$$

for all $x \in E$.

Proof. Putting $\psi(x, y)=\varphi(y)$ in inequality (3.1), then it implies (3.12). Putting $y=x$ in (3.12), we get

$$
\|f(2 x)+f(0)-2 f(x)\| \leq \varphi(x)
$$

which is the same form as condition (3.3) in the proof of Theorem 3.1, so the rest of proof runs analogously.

From Theorem 3.1 and Corollaries 3.2 and 3.3, we can obtain the following corollaries with the case $\psi(x, y)=\varphi(x)=\varphi(y)=\varepsilon$ as a natural result.

Corollary 3.4 [10]. Let E be a Banach space. Suppose that $f: G \rightarrow$ E satisfies the inequality

$$
\|f(x+y)+f(x-y)-2 f(x)\| \leq \varepsilon, \quad x, y \in G
$$

then there exists a unique additive mapping $A: G \rightarrow E$ as a solution of $(J)$ such that $A(-x)=$ $-A(x)$ and

$$
\|f(x)-f(0)-A(x)\| \leq \varepsilon, \quad x \in G
$$


12 Stability of generalized d'Alembert and Jensen equations

\section{References}

[1] R. Badora, On Hyers-Ulam stability of Wilson's functional equation, Aequationes Mathematicae 60 (2000), no. 3, 211-218.

[2] R. Badora and R. Ger, On some trigonometric functional inequalities, Functional EquationsResults and Advances, Adv. Math. (Dordr.), vol. 3, Kluwer Academic, Dordrecht, 2002, pp. 315.

[3] J. A. Baker, The stability of the cosine equation, Proceedings of the American Mathematical Society 80 (1980), no. 3, 411-416.

[4] J. A. Baker, J. Lawrence, and F. Zorzitto, The stability of the equation $f(x+y)=f(x) f(y)$, Proceedings of the American Mathematical Society 74 (1979), no. 2, 242-246.

[5] D. G. Bourgin, Approximately isometric and multiplicative transformations on continuous function rings, Duke Mathematical Journal 16 (1949), no. 2, 385-397.

[6] P. de Place Friis and H. Stetkær, On the cosine-sine functional equation on groups, Aequationes Mathematicae 64 (2002), no. 1-2, 145-164.

[7] P. Găvruța, A generalization of the Hyers-Ulam-Rassias stability of approximately additive mappings, Journal of Mathematical Analysis and Applications 184 (1994), no. 3, 431-436.

[8] Pl. Kannappan, The functional equation $f(x y)+f\left(x y^{-1}\right)=2 f(x) f(y)$ for groups, Proceedings of the American Mathematical Society 19 (1968), no. 1, 69-74.

[9] Pl. Kannappan and G. H. Kim, On the stability of the generalized cosine functional equations, Annales Academiae Paedagogicae Cracoviensis; Studia Mathematica 1 (2001), 49-58.

[10] G. H. Kim and S. H. Lee, Stability of the d'Alembert type functional equations, Nonlinear Functional Analysis \& Applications 9 (2004), no. 4, 593-604.

[11] C. T. Ng, Jensen's functional equation on groups. III, Aequationes Mathematicae 62 (2001), no. 12, 143-159.

[12] P. Sinopoulos, Functional equations on semigroups, Aequationes Mathematicae 59 (2000), no. 3, 255-261.

[13] H. Stetkær, On Jensen's functional equation on groups, Aequationes Mathematicae 66 (2003), no. $1-2,100-118$.

[14] L. Székelyhidi, The stability of d'Alembert-type functional equations, Acta Scientiarum Mathematicarum (Szeged) 44 (1982), no. 3-4, 313-320 (1983).

[15] D. Yang, The stability of Jensen's equation on amenable locally compact groups, Results in Mathematics 46 (2004), no. 3-4, 381-388.

Gwang Hui Kim: Department of Mathematics, Kangnam University, Suwon 449-702, South Korea E-mail address: ghkim@kangnam.ac.kr

Current address: School of Computer Science \& Mathematics, Victoria University, P.O. Box 14428, Melbourne City, MC 8001, Australia

Sever S. Dragomir: School of Computer Science \& Mathematics, Victoria University, P.O. Box 14428, Melbourne City, MC 8001, Australia

E-mail address: sever@csm.vu.edu.au 


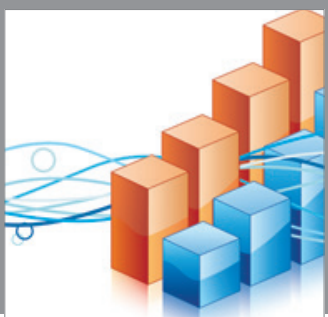

Advances in

Operations Research

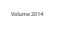

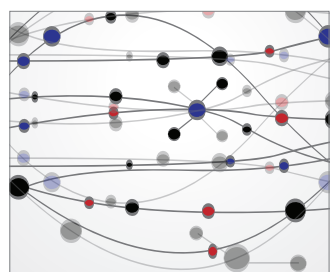

\section{The Scientific} World Journal
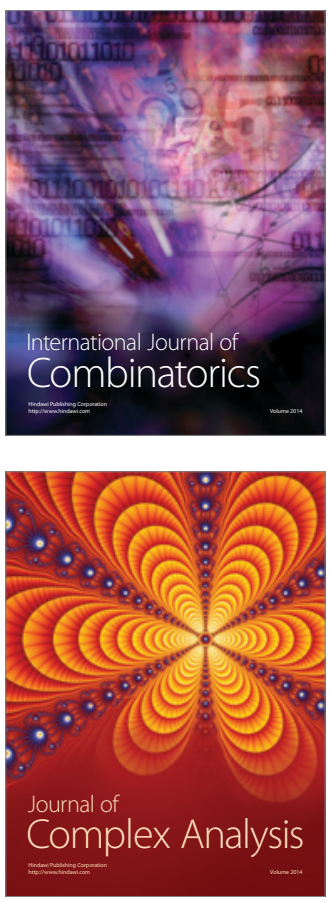

International Journal of

Mathematics and

Mathematical

Sciences
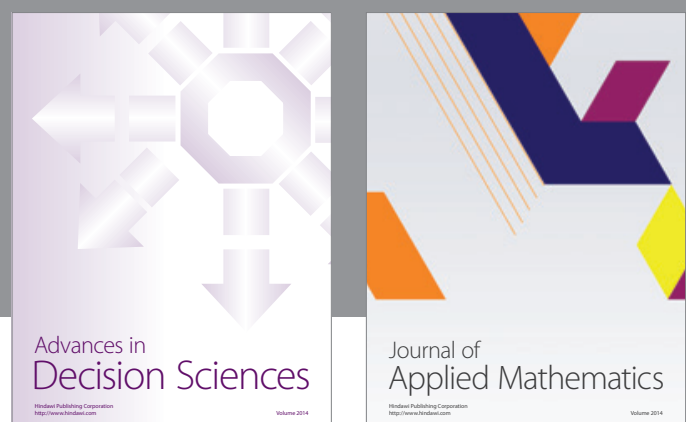

Journal of

Applied Mathematics
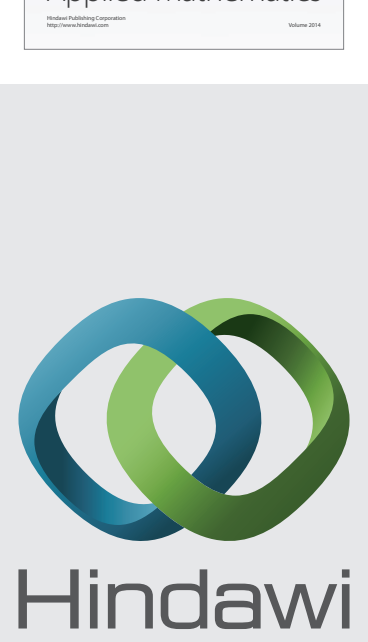

Submit your manuscripts at http://www.hindawi.com
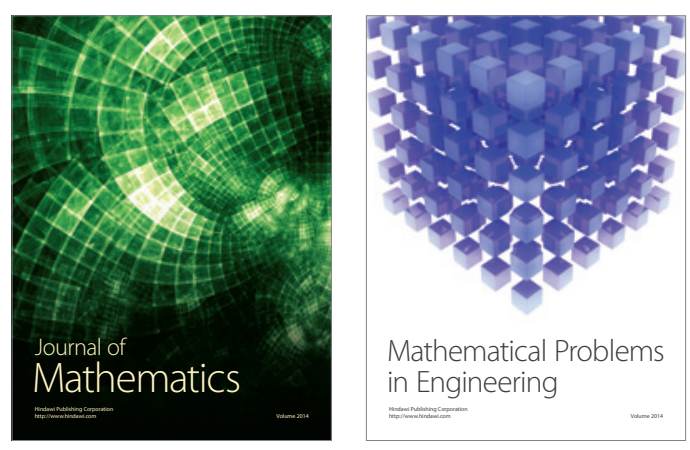

Mathematical Problems in Engineering
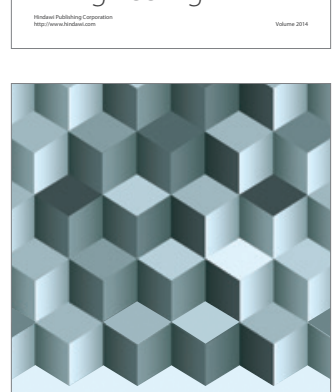

Journal of

Function Spaces
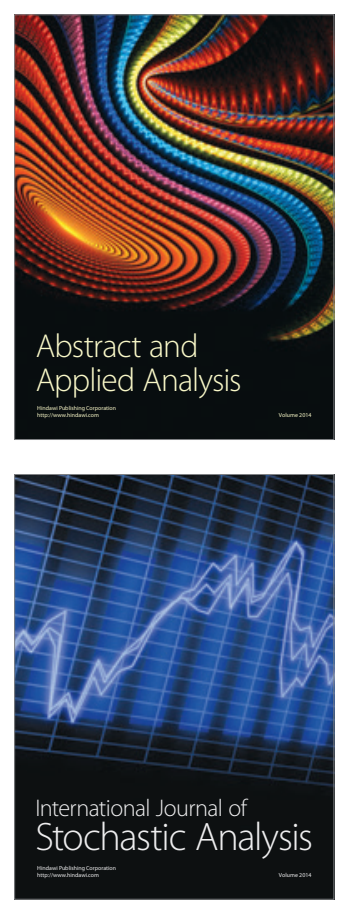

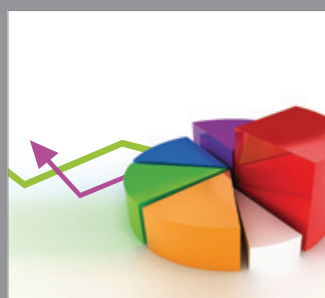

ournal of

Probability and Statistics

Promensencen
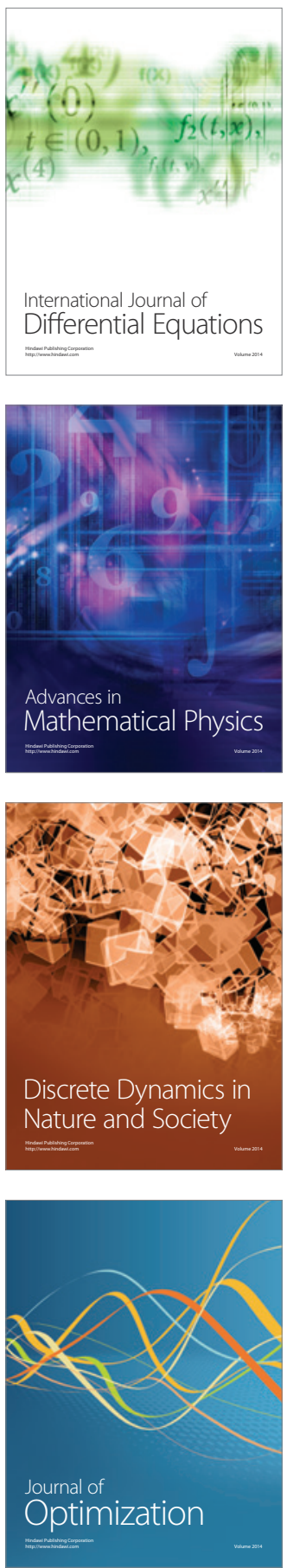